\title{
Composition of Light Solar Wind Noble Gases in the Bulk Metallic Glass flown on the Genesis
}

\section{Mission}

\author{
Journal Article \\ Author(s): \\ Grimberg, A.; Burnett, D. S.; Bochsler, P.; Baur, H.; Wieler, R. \\ Publication date: \\ 2007 \\ Permanent link: \\ https://doi.org/10.3929/ethz-b-000004575
}

Rights / license:

In Copyright - Non-Commercial Use Permitted

Originally published in:

Space Science Reviews 130(1-4), https://doi.org/10.1007/s11214-007-9150-1 


\title{
Composition of Light Solar Wind Noble Gases in the Bulk Metallic Glass flown on the Genesis Mission
}

\author{
A. Grimberg • D.S. Burnett • P. Bochsler · H. Baur • \\ R. Wieler
}

Received: 20 December 2006 / Accepted: 1 February 2007 /

Published online: 22 March 2007

(C) Springer Science+Business Media, Inc. 2007

\begin{abstract}
We discuss data of light noble gases from the solar wind implanted into a metallic glass target flown on the Genesis mission. Helium and neon isotopic compositions of the bulk solar wind trapped in this target during 887 days of exposure to the solar wind do not deviate significantly from the values in foils of the Apollo Solar Wind Composition experiments, which have been exposed for hours to days. In general, the depth profile of the $\mathrm{Ne}$ isotopic composition is similar to those often found in lunar soils, and essentially very well reproduced by ion-implantation modelling, adopting the measured velocity distribution of solar particles during the Genesis exposure and assuming a uniform isotopic composition of solar wind neon. The results confirm that contributions from high-energy particles to the solar wind fluence are negligible, which is consistent with in-situ observations. This makes the enigmatic "SEP-Ne" component, apparently present in lunar grains at relatively large depth, obsolete. ${ }^{20} \mathrm{Ne} /{ }^{22} \mathrm{Ne}$ ratios in gas trapped very near the metallic glass surface are up to $10 \%$ higher than predicted by ion implantation simulations. We attribute this superficially trapped gas to very low-speed, current-sheet-related solar wind, which has been fractionated in the corona due to inefficient Coulomb drag.
\end{abstract}

Keywords Sun: solar wind · Isotopic abundance ratios $\cdot$ Methods: laboratory

\section{Introduction}

To infer the isotopic and elemental composition of the solar photosphere from that of the solar wind ( $\mathrm{SW}$ ), it is essential to have precise information on the fractionation mechanisms

\footnotetext{
A. Grimberg $(\bowtie) \cdot$ H. Baur $\cdot$ R. Wieler

Isotope Geology, ETH Zürich, CH-8092 Zürich, Switzerland

e-mail: Grimberg@erdw.ethz.ch

D.S. Burnett

Calif. Inst. of Technology, GPS MC 100-23, Pasadena, CA 91125, USA

P. Bochsler

Physikalisches Institut, University of Bern, CH-3012 Bern, Switzerland
} 
operating at the source, i.e. the Sun and its outer convective zone, and in the solar wind itself. This is one of the major goals of NASA's Genesis mission (Burnett et al. 2003), which collected SW particles for more than $21 / 2$ years at the Lagrangian point L1 for high-precision analysis on earth. Three SW regime collector panels sampled the low-speed interstream wind, high-speed wind from coronal holes, and SW related to coronal mass ejections, respectively (Reisenfeld et al. 2007). Additional collectors sampled all SW regimes, including high-energy particles, to provide compositional information about the bulk SW and potential variations as a function of energy.

In the following we present noble gas data from a bulk metallic glass (BMG) target (Jurewicz et al. 2003) exposed to the solar corpuscular radiation during the entire exposure period. The main purpose of this experiment was to determine the dependence of the isotopic and elemental composition of light noble gases on implantation depth in order to study possible variations in their isotopic composition as a function of solar particle energy. We compare the BMG data with those from the Apollo Solar Wind Composition (SWC) experiments (Geiss et al. 2004), in situ measurements (Kallenbach et al., 1997a, 1997b, 1998) and depth profiles of the Ne isotopic composition measured in lunar samples (Black and Pepin 1969; Etique et al. 1981; Wieler et al. 1986; Benkert et al. 1993; Wieler 1998).

Noble gases are among the key elements to be studied in Genesis targets, since their composition in the Sun and the solar nebula cannot be deduced from meteorites, where they are heavily depleted. Moreover, light noble gases are relatively easy to measure in Genesis targets, and therefore are particularly well suited to study possible fractionation processes in different solar wind regimes. Precise measurements of $\mathrm{He}, \mathrm{Ne}$ and Ar isotopes in the SW have already been obtained from the SWC-foils (Geiss et al. 2004). Their short exposure duration of two days at most restricts the interpretation of the obtained data to specific SW conditions, especially to low-speed solar wind. In situ measurements, e.g. with instruments onboard the Advanced Composition Explorer (ACE) (Gloeckler et al. 1998; Mason et al. 1998; McComas et al. 1998; Smith et al. 1998) or the Solar and Heliospheric Observatory (SOHO/CELIAS) (Hovestadt et al. 1995), provide the full range of energy and regime-dependent data (Kallenbach et al. 1998; Wimmer-Schweingruber et al. 1998), but often suffer from lack of precision, which is necessary to constrain theories on isotopic fractionation in the SW. Noble gases measured in lunar regolith samples that were exposed to the SW for several million years include all SW regimes. The complicated sample history, variable losses of solar wind gases (Wielet et al. 1986, 1998; Pepin et al. 1999, 2000; Burnett et al. 2003) and a non-ideal behaviour during stepwise noble gas extraction make it difficult to deduce the bulk SW composition from such samples and to obtain reliable constraints on further parameters such as the dependence of trapped noble gas composition on implantation depth and thus particle energy.

The BMG on Genesis is well suited to deduce the composition of SW noble gases as a function of implantation depth, and hence, to possibly provide information on fractionation effects depending on the implantation energy. On the one hand, this is because the BMG etches very homogeneously in nitric acid (Heber 2002). A sufficient number of solar wind $\mathrm{Ne}$ ions were collected to analyse released gases in several tens of steps, yielding a high depth resolution. At the same time, the exposure period was short enough to prevent loss of near-surface-sited gas due to surface sputtering, as is often the case in natural (meteoritic or lunar) samples, and to minimise the production of spallogenic isotopes by galactic cosmic rays (GCR). On the other hand, the trapping behaviour for He and $\mathrm{Ne}$ irradiation in the metallic glass and other target materials was carefully tested experimentally (Grimberg et al. 
2005) and simulated with the SRIM-code (Ziegler 2004). Furthermore, precise input parameters on SW conditions during the Genesis exposure for SRIM modelling were provided by instruments onboard ACE and the Genesis spacecraft itself (Reisenfeld et al. 2007).

\section{Bulk Solar Wind He and Ne Data}

Although the BMG was primarily designed to study the dependence of trapped noble gas composition on implantation depth, we also report data on the composition of the bulk solar wind trapped by this target during the maximum activity of solar cycle 23 . Such data are important for comparison with other target materials on Genesis as well as with earlier SW collection experiments, e.g. the SWC foils or with gases trapped in lunar samples. The bulk BMG data (Table 1) were obtained by total noble gas extraction via melting of the glass or as the sum of the closed-system etching steps described below. The measured bulk isotopic ratios of $\mathrm{He}$ and $\mathrm{Ne}$ from both methods are in good agreement. To compare these data with those obtained on other target materials and to deduce absolute solar wind fluxes, one has to consider that the BMG mainly consists of transition metals $\left(\mathrm{Zr}_{58.5} \mathrm{Nb}_{2.8} \mathrm{Cu}_{15.6} \mathrm{Ni}_{12.8} \mathrm{Al}_{10.3}\right.$, subscripts in atomic-\%) with a relatively high atomic number. Since backscattering of impinging ions, the relevant process controlling the trapping efficiency of a material, depends on the atomic masses, of both, the projectile and the target, care has to be taken when comparing results from different target materials. Backscatter losses from the BMG are larger than for targets consisting of lighter elements, hence the correction is more important for the heavy BMG target than for, e.g., the SWC Alfoils. Correction factors for $\mathrm{He}$ and $\mathrm{Ne}$ isotopes listed in Table 1 have been determined

Table 1 Bulk isotopic and elemental composition from the Genesis BMG and from SWC foils reported by Geiss et al. (2004)

\begin{tabular}{|c|c|c|c|c|c|c|}
\hline Sample & ${ }^{3} \mathrm{He} /{ }^{4} \mathrm{He}\left(\times 10^{-4}\right)$ & $\pm 2 \sigma\left(\times 10^{-4}\right)$ & ${ }^{20} \mathrm{Ne} /{ }^{22} \mathrm{Ne}$ & $\pm 2 \sigma$ & ${ }^{4} \mathrm{He} /{ }^{20} \mathrm{Ne}$ & $\pm 2 \sigma$ \\
\hline \multicolumn{7}{|l|}{ Uncorr. Genesis BMG ${ }^{\mathrm{a}}$} \\
\hline CSSE, mean ${ }^{\mathrm{b}}$ & 4.34 & 0.04 & 13.64 & 0.11 & 466 & 20 \\
\hline Total Extraction, mean & 4.31 & 0.01 & 13.56 & 0.05 & 504 & 21 \\
\hline \multicolumn{7}{|l|}{ Corr. Genesis BMG ${ }^{c}$} \\
\hline CSSE, mean & 4.44 & 0.04 & 13.83 & 0.11 & 479 & 20 \\
\hline Total Extraction, mean & 4.41 & 0.01 & 13.75 & 0.05 & 518 & 21 \\
\hline \multicolumn{7}{|l|}{ SWC Foils ${ }^{\mathrm{d}}$} \\
\hline Apollo 11 & 5.38 & 0.40 & 13.50 & 1.00 & 430 & 90 \\
\hline Apollo 12 & 4.08 & 0.17 & 13.25 & 0.50 & 620 & 70 \\
\hline Apollo 14 & 4.48 & 0.28 & 13.65 & 0.40 & 550 & 70 \\
\hline Apollo 15 & 4.33 & 0.22 & 13.70 & 0.40 & 550 & 50 \\
\hline Apollo 16 & 4.42 & 0.20 & 13.80 & 0.40 & 570 & 50 \\
\hline SWC time weighted average & 4.26 & 0.22 & 13.70 & 0.30 & 570 & 70 \\
\hline
\end{tabular}

${ }^{\mathrm{a}}$ This work; mean ratios of three samples for both, CSSE and total extraction

${ }^{\mathrm{b}}$ Ratio of the integrated isotope abundances for all steps in a certain sample (12,31, and 21 steps respectively)

${ }^{\mathrm{c}}$ Backscatter correction factors for the BMG (SWC foils) are 1.024 (1.02) for ${ }^{3} \mathrm{He} /{ }^{4} \mathrm{He}, 1.014$ (1.00) for ${ }^{20} \mathrm{Ne} /{ }^{22} \mathrm{Ne}$ and 1.029 (1.12) for ${ }^{4} \mathrm{He} /{ }^{20} \mathrm{Ne}$

${ }^{d}$ Backscatter corrected values from Geiss et al. (2004), see ${ }^{c}$ for correction factors 
with implantation experiments at the CASYMS facility in Bern (Ghielmetti et al. 1983; Grimberg et al. 2005) and SRIM. For Ne, the experimental and the modelled value are consistent and we choose the SRIM value because of its smaller uncertainty. For He we adopted the CASMYS-deduced correction factor noting that SRIM simulations for the BMG seem to overestimate backscattering of very light elements in the SW energy range.

Corrected $\mathrm{He}$ and $\mathrm{Ne}$ isotopic ratios in the BMG agree well with the results of the SWC foils reported by Geiss et al. (2004). We note that the bulk SW isotopic and elemental composition of $\mathrm{He}$ and $\mathrm{Ne}$ does not vary significantly between the daily scale recorded by the SWC experiments and the 2.5 year average recorded by the Genesis BMG target. The ratios of ${ }^{4} \mathrm{He} /{ }^{20} \mathrm{Ne}$ and ${ }^{4} \mathrm{He} /{ }^{3} \mathrm{He}$ measured in different SWC experiments correlate with each other (Geiss et al. 2004), which can be explained by fractionation caused by Coulomb drag, affecting both, the elemental and the isotopic ratio simultaneously (Geiss et al. 1970; Bodmer and Bochsler 1998). The BMG data point falls right on the Coulomb drag fractionation line which is consistent with the SWC foil data (Bochsler 2007). This also confirms that even the more mobile $\mathrm{He}$ has been quantitatively retained in the $\mathrm{BMG}$ despite the estimated temperatures of $\sim 180^{\circ} \mathrm{C}$ during exposure.

\section{Depth Dependence of Trapped Solar Ne Composition in the BMG}

The mean penetration distance of ions into a target material scales with their implantation energy (Ziegler 2004). Therefore, the depth-dependent distribution of SW isotopes and elements in a material contains information about the energy-dependence of the SW composition. This information is smeared out by two effects, however. On the one hand, particles of a given implantation energy have a relatively wide depth distribution. Changes in SW velocity over a collection period result in an overlap of different depth distributions for a given particle. On the other hand, at a given moment, all SW species have similar velocities, causing heavier particles to have higher kinetic energies and thus greater penetration depths. This leads to mass fractionation upon implantation.

The depth-dependent isotopic composition of neon obtained by closed-system stepwise etching (CSSE) analyses (Heber 2002; Grimberg et al. 2005) in three BMG samples is shown in Fig. 1. In the first steps from very close to the surface, ${ }^{20} \mathrm{Ne} /{ }^{22} \mathrm{Ne}$-ratios are distinctly higher than the measured bulk SW average in the BMG of $13.75 \pm 0.05$ (Table 1) or any value reported from depth-dependent analyses of lunar grains (Black and Pepin 1969; Etique et al. 1981; Wieler et al. 1986; Benkert et al. 1993). With progressive etching the ${ }^{20} \mathrm{Ne} /{ }^{22} \mathrm{Ne}$-ratio slowly decreases and the final etching steps, releasing deeply sited particles, display ${ }^{20} \mathrm{Ne} /{ }^{22} \mathrm{Ne}-$ ratios as low as $10.5 \pm 0.3$.

Overall, the depth distribution found in the BMG is in very good agreement with SRIM simulations (Fig. 1) assuming a uniform SW composition, using the backscatter-corrected average of ${ }^{20} \mathrm{Ne} /{ }^{22} \mathrm{Ne}$ ratios determined in the total extraction analyses of $13.75 \pm 0.05$ (Table 1) and taking the velocity histogram as obtained with ACE instruments for the exposure period (Reisenfeld et al. 2007). Minor deviations of the measured data from the SRIM model predictions, showing-up in elevated ${ }^{20} \mathrm{Ne} /{ }^{22} \mathrm{Ne}$-ratios released in small gas fractions from very shallow depths in the BMG and a less steep gradient towards heavier composition with increasing depth, will be discussed in the following chapter.

The generally very good agreement of the measured depth distribution of $\mathrm{Ne}$ isotopes with SRIM predictions corroborates mass-dependent fractionation of SW ions upon implantation to be the responsible process for the observed Ne data pattern in the BMG (Grimberg et al. 2006). This process has been discussed earlier (Tamhane and Agrawal 1979; 


\section{Implantation depth according to SRIM (nm)}

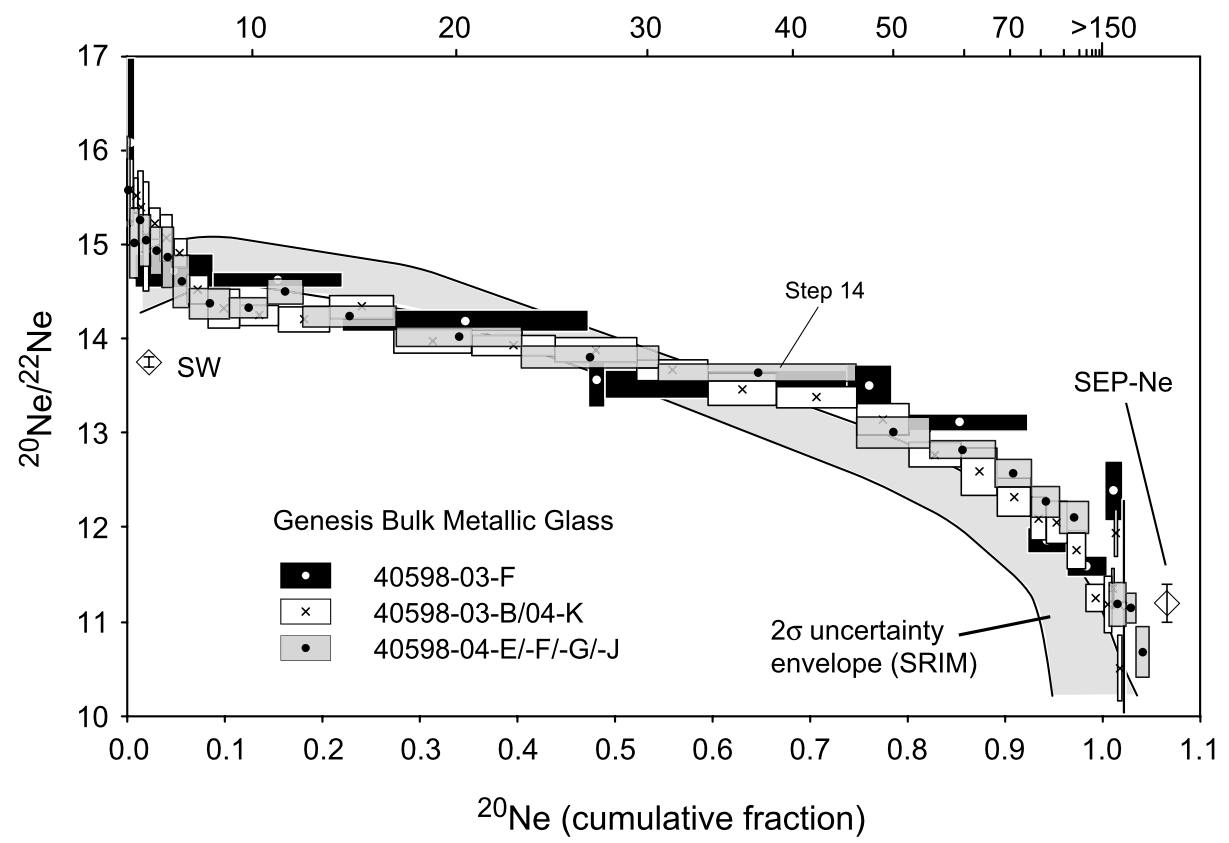

Fig. 1 Depth-dependent neon isotopic composition of implanted SW derived from three Genesis BMG samples (white, grey and black boxes) by CSSE together with SRIM simulation data (light grey shaded area in between the two solid black lines displaying the $2 \sigma$ uncertainty envelope including the statistical error and the error of the bulk measurement by total extraction, assuming a velocity-independent ${ }^{20} \mathrm{Ne} /{ }^{22} \mathrm{Ne}$ ratio of $13.75 \pm 0.05$ ), versus the cumulative ${ }^{20} \mathrm{Ne}$ fraction. Length of boxes in horizontal direction indicates gas amounts released per etching step, vertical extension of boxes indicates $2 \sigma$ uncertainties of ${ }^{20} \mathrm{Ne} /{ }^{22} \mathrm{Ne}$ ratios including ion statistics, extraction blank variability, interferences and mass discrimination. Step 14 of sample 40598-04-EFGJ had to be corrected for $\sim 23 \%$ of $\mathrm{Ne}$ contribution from a gas inclusion in the BMG opened during the etching process. ${ }^{20} \mathrm{Ne} /{ }^{22} \mathrm{Ne}$ ratios of all three BMG samples follow a trend that gets progressively heavier with depth. The implantation depth of the measured gas given on the upper abscissa is estimated from the simulated depth according to SRIM. Simulated and measured ${ }^{20} \mathrm{Ne} /{ }^{22} \mathrm{Ne}$ profiles agree very well with each other. Thus the data pattern of the BMG can be explained by a fractionation of an isotopically uniform solar wind ( $\mathrm{SW}=\mathrm{BMG}$ bulk average from total extraction) upon implantation. Remarkably, ratios in the first $8 \%$ of the gas from very close to the surface show clearly higher ${ }^{20} \mathrm{Ne} /{ }^{22} \mathrm{Ne}$ ratios than predicted by the SRIM simulations. This suggests this fraction to be very low-speed, current-sheet-related SW. The putative "SEP-Ne" data point is only for reference and should not be considered any longer.

Pepin et al. 2000; Mewaldt et al. 2001) but the solar Ne in mineral grains from lunar soils has widely been interpreted to be a mixture of two isotopically distinct components in the solar corpuscular radiation, SW-Ne near the surface, and "SEP-Ne" with a ${ }^{20} \mathrm{Ne} /{ }^{22} \mathrm{Ne}$ ratio of $11.2 \pm 0.2$ (Etique et al. 1981; Wieler et al. 1986; Benkert et al. 1993) at larger depth. The main problem with this interpretation has always been the very high required fluence of solar energetic particles (Wieler 1998; Mewaldt et al., 2001; Wimmer-Schweingruber and Bochsler 2001) since in situ analyses measured SEP/SW ratios $<0.001$, being orders of magnitude smaller than those reported from lunar samples (SEP-Ne/SW-Ne $\sim 0.1-0.4$ ). The BMG data now show unambiguously that the putative "SEP-Ne" component is not needed to explain the lunar soil data. The interpretation of the BMG data here is also 
fully consistent with the solar energetic particles fluences measured during the exposure period of Genesis with the ACE instruments SIS, CRIS and ULEIS (Mason et al. 1998; McComas et al. 1998; Stone et al. 1998a, 1998b). The integrated Ne fluence $(1 \pi)$ of suprathermal ions in the range from $5.0 \mathrm{keV} /$ nucleon to $1000 \mathrm{keV} /$ nucleon is four orders of magnitude lower than the SW fluence (SEP/SW 0.0001) for the Genesis period (Mewaldt R.A., private communication). The corresponding Ne concentrations in the BMG are much too low to be resolved from the SW contribution. The data reported here show that fractionation upon implantation is capable to produce ${ }^{20} \mathrm{Ne} /{ }^{22} \mathrm{Ne}$ ratios as low and even lower than the putative "SEP-Ne" value formerly inferred from lunar data. The "SEP"-noble gas component is thus an artefact and should not be considered any longer.

\section{Isotopic Fractionation of the Solar Wind}

As discussed above, the depth distribution of noble gas isotopes also contains, in principle, information about the fractionation operating on the SW prior to implantation, though this is complicated by implantation-induced fractionation overprinting the original SW signature. However, the initial etch steps, representing the first $8 \%$ of the Ne from very close to the BMG surface (Fig. 1), show ${ }^{20} \mathrm{Ne} /{ }^{22} \mathrm{Ne}$ ratios up to $10 \%$ higher than implied by SRIM simulations (Fig. 2). We note that losses of superficially implanted neon by diffusion would

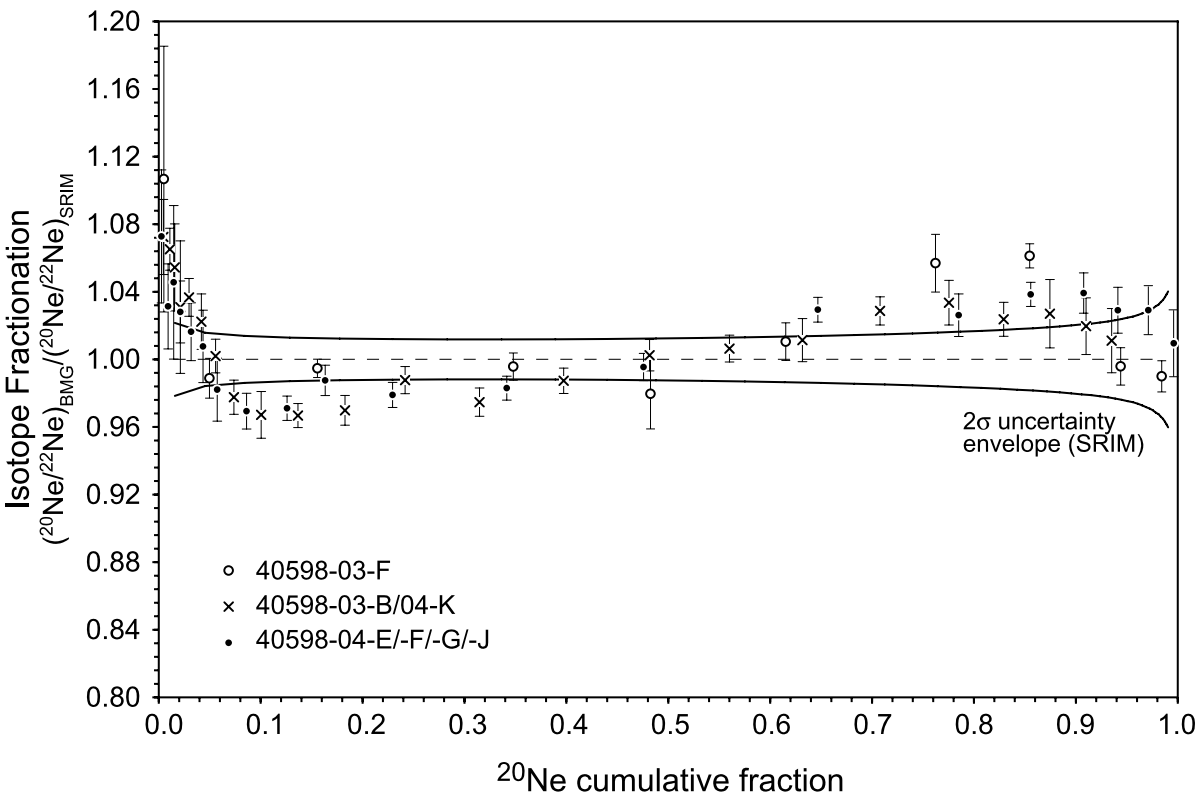

Fig. 2 Deviations of ${ }^{20} \mathrm{Ne} /{ }^{22} \mathrm{Ne}$ ratios measured in the BMG from values predicted according to SRIM (horizontal dashed line with $2 \sigma$ uncertainty envelope including the statistical error and the error of the bulk measurement by total extraction) for a uniform SW with a ${ }^{20} \mathrm{Ne} /{ }^{22} \mathrm{Ne}$ ratio of 13.75 as a function implantation depth represented by the cumulative ${ }^{20} \mathrm{Ne}$ fraction. A depletion of the heavy isotope up to a ${ }^{20} \mathrm{Ne}$ fraction of $8 \%$ can be explained by inefficient Coulomb drag according to the model of Bodmer and Bochsler (1998). The weak trend in gas released in later steps towards an enrichment of the light isotope is contrary to the Coulomb drag model as well as the findings by Kallenbach et al. (1997a, 1997b, 1998). It might indicate a not perfectly homogeneous etching rather than a fractionation of the SW at higher speed. Hence, its significance is questionable 
produce the opposite trend. The same statement applies for the effect of a possible superficial contamination of the BMG with air neon. The depletion of the heavy isotope relative to SW implanted with higher energies supports the model of isotopic fractionation due to inefficient Coulomb drag in low-speed, current-sheet-related solar wind (e.g. Wimmer-Schweingruber 1994; Bodmer and Bochsler 1998). The inefficiency of Coulomb drag in low-speed wind is corroborated by a coincident depletion of $\alpha$-particles represented by small $\mathrm{He} / \mathrm{H}$ ratios $<0.015$ (cf. Geiss et al. 1970) at low SW velocities as measured with the Genesis ion monitor. Adopting the correlation of ${ }^{4} \mathrm{He} /{ }^{20} \mathrm{Ne}$ and ${ }^{20} \mathrm{Ne} /{ }^{22} \mathrm{Ne}$ observed with the Apollo Foil experiments the Coulomb drag model (Bochsler 2007) predicts a fractionation of Ne towards a lighter isotopic composition in the low-speed SW relative to the SW average. Since the surface-near neon composition in the BMG and the Coulomb drag model are, in general, consistent we attribute the superficially trapped gas to very low-speed, current-sheet-related solar wind.

In greater depths $\left({ }^{20} \mathrm{Ne}\right.$ fraction $\left.>0.1\right)$, however, the BMG data show a slightly less steep gradient towards heavier Ne composition than predicted by the SRIM model (Fig. 1). This pattern might be explained by an enhancement of the light isotope with increasing SW energy relative to the bulk SW (Fig. 2). However, this is in contrast to in situ measurements from SOHO/CELIAS/MTOF reported by Kallenbach et al. (1997a, 1997b, 1998), that display a marginally significant enrichment of the heavy isotope by $\sim 2 \%$ over the range of $350 \mathrm{~km} / \mathrm{s}$ to $650 \mathrm{~km} / \mathrm{s}$, and it is also contradicted by any solar wind fractionation concept. The reproducibility of the three different CSSE measurements at a given implantation depth in turn limits the determination of SW fractionation prior to implantation. Hence, the significance of the trend found in greater depths $\left({ }^{20} \mathrm{Ne}\right.$ fraction $\left.>0.1\right)$ of the BMG needs further investigation; for instance some smearing of the isotopic distribution due to somewhat inhomogeneous etching cannot be ruled out at this point.

\section{Conclusions}

The isotopic composition of $\mathrm{Ne}$ as well as the He/Ne ratio of trapped solar wind in Genesis' Bulk Metallic Glass (BMG) target is fully consistent with data from the previous Apollo Solar Wind Composition experiment (Geiss et al. 2004). This shows that the BMG target quantitatively trapped and retained light noble gases from the solar wind, which is also encouraging for other Genesis targets. Thanks to the homogeneous etching of the BMG, allowing high resolution depth profiling of the Ne isotopic composition, and the detailed monitoring of SW conditions during the Genesis exposure, we have been able to show that the observed depth distribution of $\mathrm{Ne}$ isotopes is, in general, consistent with fractionation of a SW with uniform isotopic composition upon implantation. Since the measured depth profile is also similar to the distribution of solar Ne in lunar soils, an important consequence of this finding is that the putative "SEP-Ne" component is obsolete. While our data are thus consistent with a uniform SW Ne isotopic composition over most of the SW energy range, isotopically very light $\mathrm{Ne}$ representing the most shallowly implanted gas indicates that very low-speed, current-sheet related SW is fractionated relative to the bulk SW composition due to inefficient Coulomb drag. Further measurements of the He and Ar isotopic depth distribution in this target have the potential to examine the dependence of the elemental composition on SW energy.

Acknowledgements We would like to thank R.A. Mewaldt, G.M. Mason, C.M.S. Cohen, R.A. Leske, and M.E. Wiedenbeck for providing suprathermal fluence data from ACE, F. Bühler for valuable discussions, and S. Tosatti and O.J. Homan for XPS and plasma cleaning of the BMG. We also would like to thank the entire Genesis team. This work was supported by the Swiss National Science Foundation and NASA. 


\section{References}

J.P. Benkert, H. Baur, P. Signer, R. Wieler, J. Geophys. Res. - Planets 98, 13147 (1993)

D.C. Black, R.O. Pepin, Earth Planet. Sci. Lett. 6, 395 (1969)

R. Bodmer, P. Bochsler, Astron. Astrophys. 337, 921 (1998)

P. Bochsler, Space Sci. Rev. this volume (2007)

D.S. Burnett et al., Space Sci. Rev. 105, 509 (2003)

P. Etique, P. Signer, R. Wieler, Lunar Planet. Sci. Conf. XII (1981), pp. 265-267

J. Geiss, P. Hirt, H. Leutwyler, Sol. Phys. 12, 458 (1970)

J. Geiss et al., Space Sci. Rev. 110, 307 (2004)

A.G. Ghielmetti, H. Balsiger, R. Bänninger, P. Eberhardt, J. Geiss, D.T. Young, Rev. Sci. Instruments 54, 425 (1983)

G. Gloeckler et al., Space Sci. Rev. 86, 497 (1998)

A. Grimberg, F. Bühler, P. Bochsler, V.S. Heber, H. Baur, R. Wieler, Lunar Planet. Sci. XXXVI, abstract \#1355 (2005)

A. Grimberg et al., Science 314, 1133 (2006)

V. Heber, PhD Thesis, ETH Zürich, Nr. 14579, 2002, p. 157

D. Hovestadt et al., Sol. Phys. 162, 441 (1995)

A.J.G. Jurewicz et al., Space Sci. Rev. 105, 535 (2003)

R. Kallenbach et al., J. Geophys. Res. - Space Phys. 102, 26895 (1997a)

R. Kallenbach et al., in 31st ESALAB Symposium, Correlated Phenomena at the Sun, in the Heliosphere and in Geospace (1997b), pp. 33

R. Kallenbach et al., Space Sci. Rev. 85, 357 (1998)

G.M. Mason et al., Space Sci. Rev. 86, 409 (1998)

D.J. McComas et al., Space Sci. Rev. 86, 563 (1998)

R.A. Mewaldt, R.C. Ogliore, G. Gloeckler, G.M. Mason, in R. Wimmer-Schweingruber (ed.), Solar and galactic composition. AIP Conf. Proc. 598, 393-398 (2001)

R.O. Pepin, R.H. Becker, D.J. Schlutter, Geochimica et Cosmochimica Acta 63, 2145 (1999)

R.O. Pepin, R.L. Palma, D.J. Schlutter, Meteorit. Planet. Sci. 35, 495 (2000)

D.B. Reisenfeld et al., Space Sci. Rev. this volume (2007)

C.W. Smith et al., Space Sci. Rev. 86, 613 (1998)

E.C. Stone et al., Space Sci. Rev. 86, 357 (1998a)

E.C. Stone et al., Space Sci. Rev. 96, 285 (1998b)

A.S. Tamhane, J.K. Agrawal, Earth Planet. Sci. Lett. 42, 243 (1979)

R. Wieler, Space Sci. Rev. 85, 303 (1998)

R. Wieler, H. Baur, P. Signer, Geochimica et Cosmochimica Acta 50, 1997 (1986)

R.F. Wimmer-Schweingruber, PhD Thesis, University Bern, 1994

R.F. Wimmer-Schweingruber, P. Bochsler, O. Kern, G. Gloeckler, J. Geophys. Res. 103(A9), 20621 (1998)

R.F. Wimmer-Schweingruber, P. Bochsler, in Solar and Galactic Composition. AIP Conf. Proc., ed. by R. Wimmer-Schweingruber, vol. 598 (2001), p. 399

J.F. Ziegler, Nucl. Instruments Methods Phys. Res. Sect. B 219(20), 1027 (2004) 